\title{
Analysis of the ICME on 24 August 2001
}

\author{
Nedelia A. Popescu ${ }^{1}$ \\ ${ }^{1}$ Astronomical Institute of Romanian Academy, Bucharest, RO-040557, Romania \\ email: nedelia@aira.astro.ro
}

\begin{abstract}
The analysis of in-situ data recorded by Ulysses for the ICME on 24 August 2001 is carried out, using different signatures such as $\mathrm{He}^{++}$abundance enhancement, low ion temperature, low velocity, and anomalies of abundance and charge state of heavy ion species.
\end{abstract}

Keywords. heliosphere, ICME signatures

\section{Introduction}

In this paper we present detailed description and analysis of the ICME on 24 August 2001 by means of classical identification of ICMEs $\left(\mathrm{He}^{++}\right.$abundance enhancement, low kinetic temperature, low velocity); plasma dynamics signatures (thermal index $I_{t h}>1$ ); plasma composition signatures (anomalies of abundance and charge state of heavy ion species, low ion temperature and velocities).

\section{24 August 2001 ICME signatures analysis}

The duration of the ICME on 24 August 2001 is 12 hours, between 24 August-13:00 UT, and 25 August-01:00 UT. In order to analyze the characteristic signatures of an ICME we consider the in situ data recorded by Ulysses/SWOOPS and SWICS.

\subsection{Classical identification of ICMEs}

a) The ICME location is determined by the high helium abundance enhancement, that is greater than 0.08 (Neugebauer \& Goldstein 1997). In Fig. 1 solid lines denote the borders of the studied ICME.

b) Both proton and electron temperatures tend to be lower within an ICME, in comparison with the temperature of surrounding solar wind. Richardson et al. (1997) proposed as an indicator of an ICME the condition: $T_{e} / T_{p}>2$. This condition is fulfilled by the studied ICME on 24 August 2001 (Fig. 2).

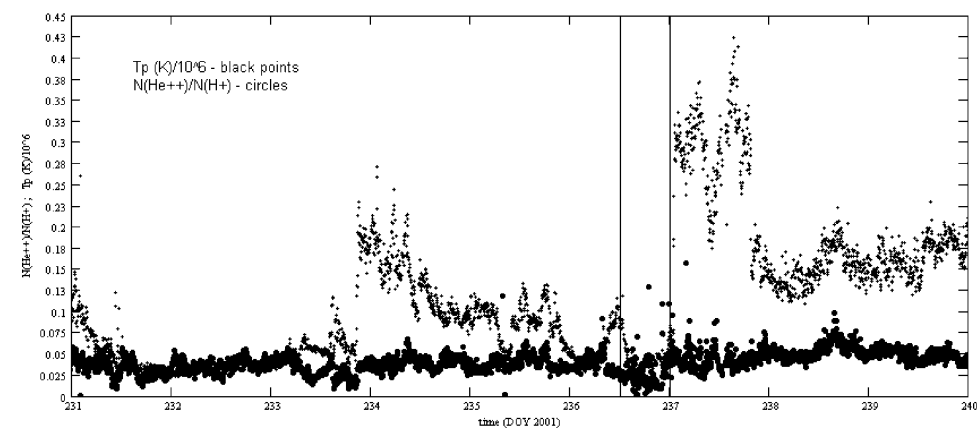

Figure 1. Alpha $\left(\mathrm{He}^{++}\right)$abundance distribution and proton temperature distribution. 


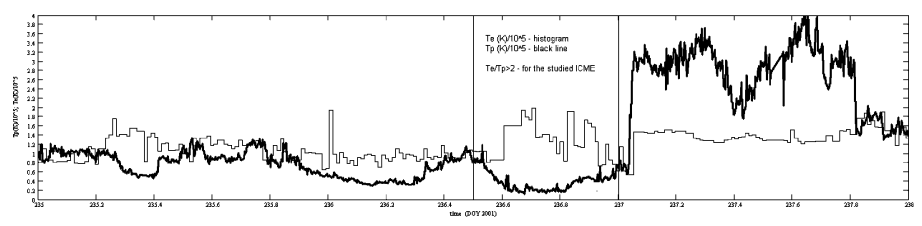

Figure 2. Proton and electron temperatures distributions.

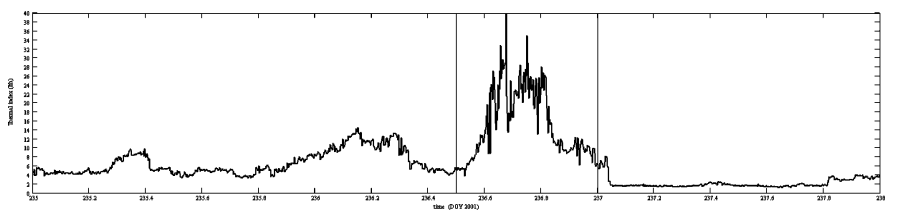

Figure 3. Thermal index $I_{t h}$ distribution.

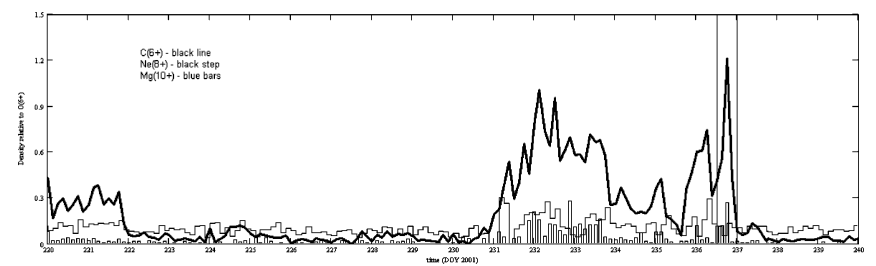

Figure 4. $\mathrm{C}(6+), \mathrm{Ne}(8+), \mathrm{Mg}(10+)$ densities relative to $\mathrm{O}(6+)$ distributions.

\subsection{Plasma dynamics signature}

Neugebauer \& Goldstein (1997) defined the thermal index as follows: $I_{t h}=\left(500 V_{p}+\right.$ $\left.1.75 \times 10^{5}\right) / T_{p}$, where $T_{p}=$ plasma proton temperature; $V_{p}=$ plasma proton velocity. If $I_{t h}>1$, plasma seems to be associated with an ICME (Fig. 3).

\subsection{Plasma composition signatures}

1) Enhancement of $\mathrm{C}(6+), \mathrm{Ne}(8+), \mathrm{Mg}(10+)$ densities relative to $\mathrm{O}(6+)$ (see Fig. 4).

2) Criteria of low ion temperature: In Fig. 5 the temperatures distributions for different heavy ions are presented. In the studied interval all temperatures are low (SWICS data). From Fig. 1 the proton temperature depression can be observed too (SWOOPS data).

3) Heavy ion species present anomalies of abundance and charge state:

a) The optimum threshold value for the average Fe charge state is considered < $Q F e>=11$ (Lepri \& Zurbuchen 2004). Greater values are indicators of the presence of ICMEs. The distributions of $\mathrm{Fe}$ average charge state and the $\mathrm{Fe} / \mathrm{O}$ abundance are presented in Fig. 6 (top panel). In the studied interval, the value of average charge state is between 10.5 and 11 and $\mathrm{Fe} / \mathrm{O}<2$.

b) The enhanced charge-state ratios $C(6+) / C(5+)$ and $O(7+) / O(6+)$ can be observed in Fig. 6 (bottom panel). The threshold $O(7+) / O(6+)=0.8$ corresponds to a freezing-in temperature of $2.05 \times 10^{6} \mathrm{~K}$, this condition is satisfied in the studied case. This result is in good agreement with the velocity distribution (in the studied interval of time the velocity is $\sim 550 \mathrm{~km} / \mathrm{s}$ ). 

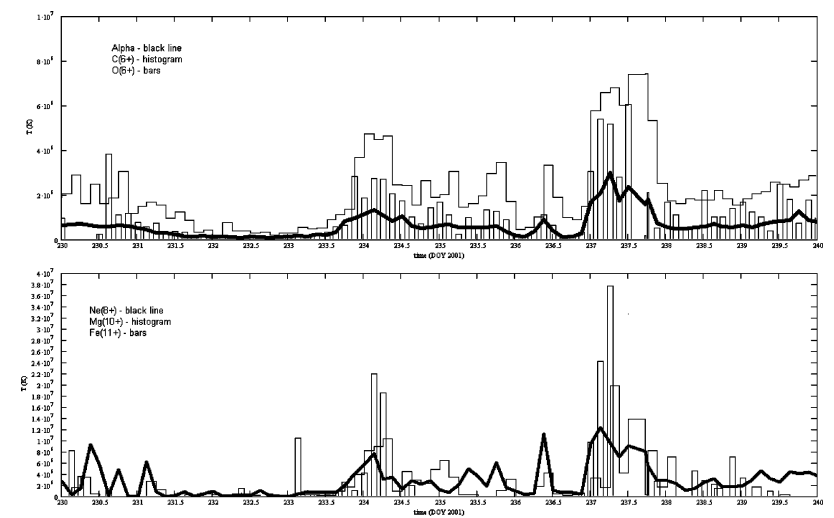

Figure 5. The temperatures distributions for different heavy ions.
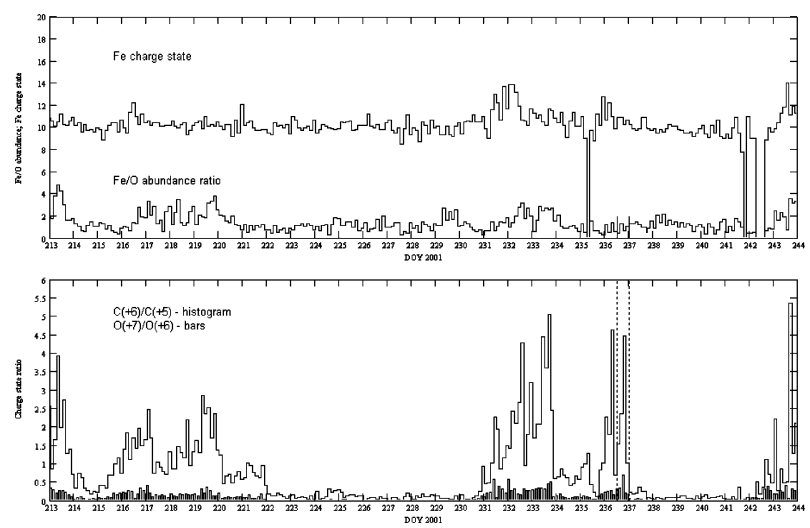

Figure 6. $\mathrm{Fe}$ average charge state and the $\mathrm{Fe} / \mathrm{O}$ abundance distribution (top panel); Charge-state ratios $C(6+) / C(5+)$ and $O(7+) / O(6+)$ (bottom panel)

\section{Conclusions}

The ICME on 24 August 2001 verifies almost all classical identification conditions, plasma dynamics and composition signatures. These signatures are not necessarily present simultaneously and define exact the same region of the solar wind. Few signatures verify only the threshold values $\left(N\left(H e^{++}\right) / N\left(H^{+}\right)=0.08, O(7+) / O(6+)=0.8\right)$, but other signatures fulfilled the imposed condition for the detection of an ICME. This analysis has been done in order to follow the interplanetary trajectory of some coronal mass ejections, starting from Ulysses satellite back to the Sun. We suspect this ICME to be the interplanetary counterpart of the 14-15 August 2001 solar event (Oncica, Popescu, \& Dumitrache 2008).

\section{References}

Lepri, S. T. \& Zurbuchen, T. H. 2004, J. Geophys. Res., 105, 29, 231

Neugebauer, M. \& Goldstein, R. 1997, in: N. Crooker et al. (eds.), Coronal Mass Ejections, AGU, p. 245

Oncica, A., Popescu, N., \& Dumitrache, C. 2008, in: V.Mioc et al. (eds), Exploring the Solar System and the Universe, AIP Conference Proceedings, 1043, 323

Richardson, I. G., Farrugia, C. J., \& Cane, H. V. 1997, J. Geophys. Res., 102, 4691 